\title{
Target Fragmentation in Semi-Inclusive DIS: Fracture Functions, Cut Vertices \& the OPE
}

\author{
M. Grazzini \\ Institute for Theoretical Physics, ETH-Hönggerberg, \\ 8093 Zürich, Switzerland. \\ E-mail: grazzini@itp.phys.ethz.ch \\ G. M. Shore \\ TH Division, CERN, \\ CH-1211 Genève 23, Switzerland. \\ and \\ Physics Department, University of Wales Swansea, \\ Singleton Park, Swansea SA2 8PP, UK. \\ E-mail: g.m.shore@swansea.ac.uk \\ B. E. White \\ Physics Department, University of Wales Swansea, \\ Singleton Park, Swansea SA2 8PP, UK. \\ E-mail: pywhite@python.swan.ac.uk
}

\begin{abstract}
We discuss semi-inclusive Deep Inelastic Scattering (DIS) in the $z \rightarrow 1$ limit, in particular the relationship between fracture functions, generalised cut vertices and Green functions of the composite operators arising in the OPE. The implications, in the spin-polarised case, for testing whether the "proton spin" effect is targetindependent are explored. Explicit calculations in $\left(\phi^{3}\right)_{6}$ theory are presented which are consistent with our observations.
\end{abstract}

CERN-TH/99-90

ETH-TH/99-07

SWAT/99-223 - March 1999 


\section{Introduction}

The "proton spin" problem [1] concerns the anomalous suppression of the Ellis-Jaffe sum rule for the first moment $\Gamma_{1}^{p}$ of the polarised proton structure function $g_{1}$. In a series of papers, Narison, Shore and Veneziano $[2,3,4]$ have shown that the underlying mechanism can be understood as topological charge screening. The flavour singlet contribution to the sum-rule, far from being a measure of quark or gluon spins, is in fact a measure of gluon topological charge. The screening is a generic property of the QCD vacuum rather than a special property of the proton target.

This interpretation suggests that the "proton spin" suppression is in fact a targetindependent phenomenon, and recently [5] it has been proposed to test this directly in polarised, semi-inclusive DIS, where we sum over all possible final states containing a hadron $h$. In the $z \rightarrow 1$ limit, where $z$ is the fraction of the proton momentum carried by $h$, [fig. 2a] the polarised, semi-inclusive structure function $\Delta M^{h / p}$ is modelled by having the property of Regge factorisation into a sum over products of $\mathrm{p}$-h- $\mathcal{R}$ couplings and polarised structure functions of the Regge exchanges $g_{1}^{\mathcal{R}}$. By comparing experiments with different targets $N=p, n$ and different tagged hadrons $h$, measurements of $\Delta M^{h / N}$ at $z \rightarrow 1$ can give ratios of the first moments of $g_{1}^{\mathcal{R}}$ for reggeons $\mathcal{R}$ with different $\mathrm{SU}(3)$ flavour quantum numbers. According to the target-independence conjecture, these ratios can be predicted simply in terms of SU(3) group theoretic factors and a universal suppression factor determined by the proton spin data.

A key requirement of the analysis of $[2,3,4]$ was the identification of the structure function with the matrix element of a local composite operator arising in the OPE, and the subsequent decomposition of the matrix element into a composite operator propagator and the corresponding "1PI" vertex function. In order to provide a rigorous theoretical foundation to the proposal of [5] in the semi-inclusive case, we need to show that a similar decomposition is valid.

In inclusive DIS, where we sum over all possible final hadronic states, the structure functions factorise into hard, perturbatively calculable factors and parton distribution functions, which parametrise the non-perturbative physics:

\section{Structure function $=$ (Hard physics $) \otimes$ (Parton dist. function)}

The moments of the parton distributions can be represented either as matrix elements of local operators or as space-like cut vertices $[6,7,8,9,10]$. It has been shown explicitly $[8,10]$ that these representations are equivalent, i.e. in the inclusive case, the cut vertices and local operator Green functions are identical.

In the case of semi-inclusive DIS in the target fragmentation region where $h$ has little transverse momentum relative to the incoming proton, the semi-inclusive structure 
functions also factorise into hard physics and fracture functions $[11,12,13,14,15]$ : [fig. 1]

Semi-incl. struct. fn. $=$ (Hard physics) $\otimes$ (Fracture function)

The fracture functions give the joint probability distribution for finding a parton of specified type and a hadron $h$ in the proton. Just as for the usual parton distributions, they absorb all the infra-red singularities. Recently, it was shown [14] that (extended) fracture functions can be represented as generalised space-like cut vertices. However, these generalised cut vertices cannot be identified with local operator matrix elements; the familiar OPE analysis of inclusive DIS is not directly applicable in the case of semi-inclusive DIS.

We can therefore summarise the relationships between the various objects as follows:

$$
\text { Parton distributions } \stackrel{\text { moments }}{\longleftrightarrow} \text { Cut vertices }=\text { Green functions }
$$

Fracture functions $\stackrel{\text { moments }}{\longleftrightarrow}$ Generalised cut vertices $\neq$ Green functions

At first sight, it therefore appears that the composite operator propagator - 1PI vertex methods of $[3,4]$ are not applicable to semi-inclusive DIS. This is, however, only partially true. The purpose of this paper is to examine precisely the relationship between fracture functions, generalised cut vertices and Green functions, and to determine under what circumstances the methods of $[3,4]$ can be applied to semi-inclusive DIS. We will present evidence that the generalised cut vertices simplify sufficiently in the $z \rightarrow 1$ limit to allow the factorisation of the same composite operator propagator from the semi-inclusive structure function as in the inclusive case. This supports the proposal in ref.[5] to test target independence and universal topological charge screening in semi-inclusive DIS in the $z \rightarrow 1$ limit.

To make this assertion plausible, we have performed perturbative calculations of generalised cut vertices to one loop in a simplified model expected to share many features (e.g. asymptotic freedom, collinear singularities, etc.) of perturbative QCD, viz. massless $\phi^{3}$ theory in six dimensions. We find that, in the $z \rightarrow 1$ limit, the dominant diagrams have such a simple analytic structure that they can indeed be expressed as Green functions in the same way as the inclusive case.

This paper is organised as follows: In section 2, we summarise the "proton spin" effect; section 3 provides definitions of kinematic variables for semi-inclusive DIS and an overview of factorisation of hard processes; our proof of the relation of generalised cut vertices and Green functions is in section 4; section 5 contains the results of the perturbative calculations of the generalised cut vertices. Our conclusions and discussion of the implications of these results are given in section 6 . 

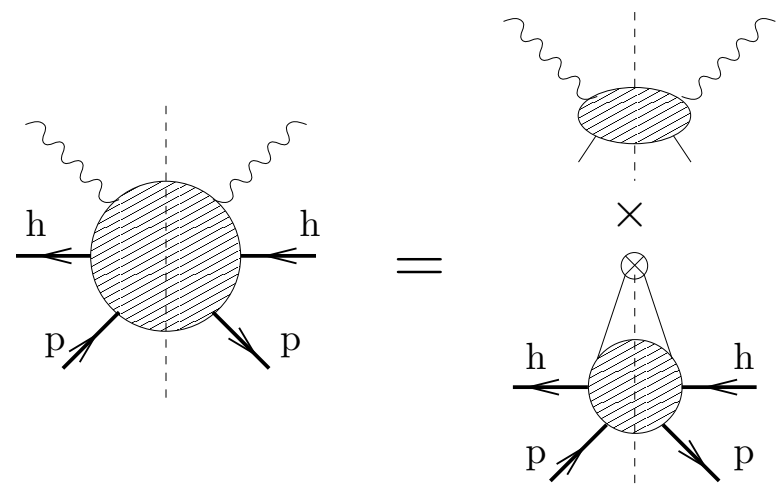

Figure 1: Factorisation of the semi-inclusive structure functions. The blob on the bottom right is the fracture function.

\section{Local Operators and Proper Vertices}

In this section we summarise the resolution of the EMC/SMC "proton spin" problem proposed in refs. $[2,3,4,16]$ in terms of gluon topological charge screening. We emphasise the need to express the target distribution functions in terms of matrix elements of local operators.

The measurement of the first moment $\Gamma_{1}^{p}$ of the polarised proton structure function by the EMC and SMC collaborations has shown it to be suppressed relative to its OZI expectation. In other words, $a^{0}$ is suppressed compared with $a^{8}$, where $a^{0}$ and $a^{8}$ appear in the OPE of $\Gamma_{1}^{p}$ :

$$
\Gamma_{1}^{p}=\int_{0}^{1} d x g_{1}^{p}\left(x, Q^{2}\right)=\frac{1}{12} C_{1}^{\mathrm{NS}}\left(\alpha_{s}\right)\left(a^{3}+\frac{1}{3} a^{8}\right)+\frac{1}{9} C_{1}^{\mathrm{S}}\left(\alpha_{s}\right) a^{0}\left(Q^{2}\right),
$$

where

$$
\begin{gathered}
\left\langle p ; s\left|A_{\mu}^{3}\right| p ; s\right\rangle=\frac{1}{2} a^{3} s_{\mu}, \quad\left\langle p ; s\left|A_{\mu}^{8}\right| p ; s\right\rangle=\frac{1}{2 \sqrt{3}} a^{8} s_{\mu}, \\
\left\langle p ; s\left|A_{\mu}^{0}\right| p ; s\right\rangle=a^{0}\left(Q^{2}\right) s_{\mu},
\end{gathered}
$$

with $s_{\mu}=\bar{u}_{s}(p) \gamma_{\mu} \gamma_{5} u_{s}(p)$ being the proton polarisation vector. In the QCD parton model $[17,18]$

$$
\begin{gathered}
a^{3}=\Delta u-\Delta d, \quad a^{8}=\Delta u+\Delta d-2 \Delta s, \\
a^{0}\left(Q^{2}\right)=\Delta u+\Delta d+\Delta s-n_{f} \frac{\alpha_{s}}{2 \pi} \Delta g\left(Q^{2}\right) .
\end{gathered}
$$

$n_{f}$ is the number of quark flavours, while $\Delta u, \Delta d, \Delta s$ and $\Delta g$ are the first moments of the polarisation asymmetries of the parton distributions.

The OZI expectation is given by approximating $\Delta s \simeq 0 \simeq \Delta g$, so that $a^{0} \simeq a^{8}$. There are two important points:

1. The form factors $a^{0}, a^{3}$ and $a^{8}$ are (reduced) matrix elements of local, composite operators. 
2. $a^{0}\left(Q^{2}\right)$ depends on the hard scale $Q^{2}$ through the term proportional to $\Delta g\left(Q^{2}\right)$. This is a result of the chiral $U_{A}(1)$ anomaly. This is already an indication that $a^{0}$ is not to be interpreted as a measurement of spin in the QCD parton model $[16,19]$.

One can explain the OZI violations in the following way. Because of the $U_{A}(1)$ anomaly, the flavour singlet axial current $A_{\mu}^{0}$ obeys an anomalous Ward identity written, neglecting quark masses,

$$
\partial^{\mu} A_{\mu}^{0}-2 n_{f} Q \simeq 0
$$

where $Q=\frac{\alpha_{s}}{8 \pi} \operatorname{tr} G_{\mu \nu} \widetilde{G}^{\mu \nu}$ is the topological charge density. Using (4), one can express

$$
a^{0}\left(Q^{2}\right)=\frac{1}{2 M_{p}} 2 n_{f}\langle p|Q| p\rangle .
$$

Thus, $a^{0}$ measures topological charge rather than parton spin. The suppression of $a^{0}$ is due to topological charge screening by the QCD vacuum. We can see this in terms of the external leg propagators of the operator $Q$; the rhs. of (5) can be decomposed into zero-momentum propagators and $1 \mathrm{PI}$ vertices $\Gamma^{1 \mathrm{PI}}$, so that

$$
\begin{aligned}
a^{0}\left(Q^{2}\right) & =\frac{1}{2 M_{p}} 2 n_{f}\left[\langle 0|T(Q Q)| 0\rangle \Gamma_{Q \mathrm{pp}}^{1 \mathrm{PI}}+\left\langle 0\left|T\left(Q \Phi_{5}\right)\right| 0\right\rangle \Gamma_{\Phi_{5} \mathrm{pp}}^{1 \mathrm{PI}}\right] \\
& =\frac{1}{2 M_{p}} 2 n_{f}\left[\chi(0) \Gamma_{Q \mathrm{pp}}^{1 \mathrm{PI}}+\sqrt{\chi^{\prime}(0)} \Gamma_{\Phi_{5} \mathrm{pp}}^{1 \mathrm{PI}}\right]
\end{aligned}
$$

where $\Phi_{5}$ is proportional [2] to $\bar{q} \gamma_{5} q$ and

$$
\chi\left(k^{2}\right)=i \int d^{d} x e^{i k \cdot x}\langle 0|T(Q(x) Q(0))| 0\rangle, \quad \chi^{\prime}(0)=\left.\frac{d}{d k^{2}} \chi\left(k^{2}\right)\right|_{k^{2}=0} .
$$

From the chiral Ward identities, it can be shown that $\chi(0)$ vanishes for zero quark mass. Performing a similar decomposition for $a^{8}$ results in a Goldberger-Treiman relation, in terms of $\Gamma_{\Phi_{5}^{8} \mathrm{pp}}^{1 \mathrm{PI}}$, for the flavour octet axial charge in the chiral limit. With the assumption, justified in refs. $[2,3,4]$, that the RG invariant $1 \mathrm{PI}$ vertices $\Gamma_{\Phi_{5}^{8} \mathrm{Pp}}^{\mathrm{PI}}$ satisfy the OZI rule, we find that

$$
\frac{a^{0}\left(Q^{2}\right)}{a^{8}}=\left.\frac{\sqrt{6}}{f_{\pi}} \sqrt{\chi^{\prime}(0)}\right|_{Q^{2}} .
$$

In this proposal, the suppression in $a^{0}\left(Q^{2}\right)$ is due to an anomalously small value of the slope of the topological susceptibility $\chi^{\prime}(0)$ compared with its OZI expectation $f_{\pi}^{2} / 6 . \chi^{\prime}(0)$ has been estimated using QCD spectral sum-rule techniques [3, 4] and the results are in good agreement with the measured suppression.

We emphasise the following aspects of the analysis: 


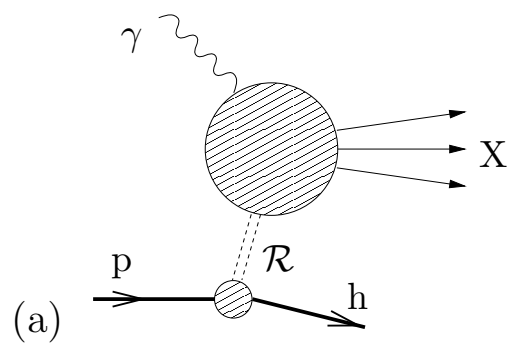

(b)

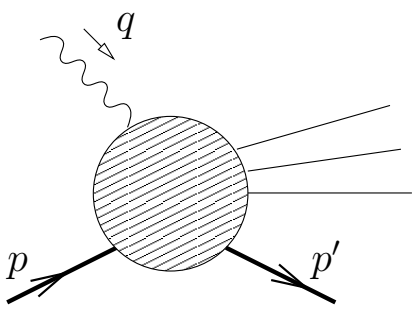

Figure 2: (a) Amplitude in the $z \rightarrow 1$ limit. Proton $p$ couples to hard hadron $h$ and reggeon $\mathcal{R}$. (b) Definitions of momenta for semi-inclusive DIS.

1. The ratio $a^{0} / a^{8}$ does not depend on the target species; the only references to "proton" were contained in the 1 PI vertices $\Gamma^{1 \mathrm{PI}}$.

2. The procedure of extracting the propagators was dependent crucially upon expressing $\Gamma_{1}^{p}$ in terms of matrix elements or Green functions of local, composite operators.

It is then natural to ask whether we can test this target-independence conjecture directly. One possibility is to measure the polarised structure functions of Regge poles in the $-t / Q^{2} \ll 1, z \rightarrow 1$ limit of polarised, semi-inclusive DIS. [fig. 2a] We can model (cf. ref. [21]) the polarised, semi-inclusive structure function for that process $\Delta M^{h / p}\left(Q^{2}, \bar{x}, z, t\right)$ by

$$
\Delta M^{h / p}\left(Q^{2}, \bar{x}, z, t\right) \stackrel{z \rightarrow 1}{\longrightarrow} F(t)(1-z)^{-2 \alpha_{\mathcal{R}}(t)} g_{1}^{\mathcal{R}}\left(Q^{2}, \bar{x}, t\right),
$$

where $h$ is the hard, final state hadron and $g_{1}^{\mathcal{R}}$ is the reggeon $\mathcal{R}$ 's polarised structure function. (The kinematic variables are defined in section 3.) By taking ratios of $\Delta M^{h / N}$ for different tagged hadrons $h$ and nucleon targets $N=p, n$, we can in effect measure ratios of the $g_{1}^{\mathcal{R}}$ for reggeons with different $\mathrm{SU}(3)$ flavour quantum numbers. For example, if we choose $h$ to be a $\pi^{-}$, then there must be an exchange with the the quantum numbers of a $\Delta^{++}$. According to the target independence conjecture, these ratios will depend only on SU(3) Clebsch-Gordon factors and a universal suppression factor which can be extracted from the inclusive "proton spin" measurement.

In what follows, we will discuss whether such quantities as $g_{1}^{\mathcal{R}}$ depend on the insertion of a local operator in the $z \rightarrow 1$ limit, as required if their flavour singlet first moments are indeed to be governed by the slope of the topological susceptibility $\chi^{\prime}(0)$.

\section{Inclusive \& Semi-Inclusive DIS}

Here we define inclusive and semi-inclusive structure functions for the simpler situation of scalar field theory, and we remind the reader how the factorisation of the hard physics is expressed. 
We define the inclusive structure function as

$$
W(q, p)=\frac{Q^{2}}{2 \pi} \sum_{X} \int d^{d} x e^{i q \cdot x}\langle p|j(x)| X\rangle\langle X|j(0)| p\rangle,
$$

where $j(x)=\phi^{2}(x)$ plays the role of the electromagnetic current ${ }^{1}$ and the sum is over all possible final states. $p$ and $q$ are the momenta of the "proton" and "photon" respectively. Factorisation of the hard physics is written as

$$
W\left(Q^{2}, x\right)=\int_{x}^{1} \frac{d u}{u} f(u) C\left(\frac{x}{u}, Q^{2}\right)
$$

where $Q^{2}=-q^{2}$ and $x=Q^{2} / 2 p \cdot q . C$ is calculable perturbatively and $f$ is a "parton" distribution function, parametrising the non-perturbative physics. Taking moments wrt. $x$, we have

$$
\int_{0}^{1} d x x^{j-1} W\left(Q^{2}, x\right)=f^{j} C^{j}\left(Q^{2}\right)
$$

where $f^{j}$ and $C^{j}$ are $j$ th moments of $f$ and $C . f^{j}$ can be represented either as a Green function of a composite operator or as a space-like cut vertex. (Section 4.)

To define the semi-inclusive structure function, we constrain there to be a hadron $h$ of a specified type and momentum $p^{\prime}$ in the final state: [fig. $2 \mathrm{~b}$ ]

$$
W\left(q, p, p^{\prime}\right)=\frac{Q^{2}}{2 \pi} \sum_{X} \int d^{d} x e^{i q \cdot x}\langle p|j(x)| h, X\rangle\langle h, X|j(0)| p\rangle,
$$

The convenient Lorentz-invariant variables for this process are as follows:

$$
\begin{gathered}
Q^{2}=-q^{2}, \quad t=\left(p-p^{\prime}\right)^{2}, \\
z=\frac{p^{\prime} \cdot q}{p \cdot q}, \quad x=\frac{Q^{2}}{2 p \cdot q} .
\end{gathered}
$$

We also use

$$
\bar{x}=\frac{Q^{2}}{2\left(p-p^{\prime}\right) \cdot q}=\frac{x}{1-z} .
$$

There are two, distinct contributions to the process, distinguished by the value of $t$, namely the target fragmentation region at small $t$ and the current fragmentation region at large $t$, so that $W=W_{\text {curr }}+W_{\text {targ. }}$. In the target fragmentation region we may neglect quantities suppressed by powers of $t / Q^{2}$.

\footnotetext{
${ }^{1}$ There is no need to decompose $W$ into Lorentz scalar structure functions since it already is a Lorentz scalar.
} 
The target fragmentation contributions can be handled by introducing extended fracture functions $\mathcal{M}(x, z, t)^{2}$ to parametrise the non-perturbative physics, so that ${ }^{3}$

$$
W_{\operatorname{targ}}\left(Q^{2}, \bar{x}, z, t\right)=\int_{\bar{x}}^{1} \frac{d u}{u} \mathcal{M}(u, z, t) C\left(\frac{\bar{x}}{u}, Q^{2}\right),
$$

where $C$ are calculable perturbatively. It has recently been shown that the moments of (extended) fracture functions can be represented as generalised cut vertices [14]. (Section 4.) This is the first step in the chain of identifications described in the introduction amongst fracture functions, cut vertices and Green functions, and is central to our analysis.

Taking the moment with respect to $\bar{x}$ of (16) we get

$$
\int_{0}^{1} d \bar{x} \bar{x}^{j-1} W_{\operatorname{targ}}\left(Q^{2}, \bar{x}, z, t\right)=\mathcal{M}^{j}(z, t) C^{j}\left(Q^{2}\right)
$$

where $\mathcal{M}^{j}$ and $C^{j}$ are $j$ th moments of $\mathcal{M}$ and $C$ respectively. (17) generalises the leading twist part of the OPE to semi-inclusive processes, with $\mathcal{M}^{j}$ given by the lowest twist generalised cut vertex.

Specialising further to the régime of interest, we consider the $z \rightarrow 1$ limit. [fig. 2a] In the context of QCD, this has been modelled with reggeon exchanges, with some success in describing data [21]. The asymptotic behaviour of $\mathcal{M}$ would be

$$
\mathcal{M}(\bar{x}, z, t) \simeq F(t)(1-z)^{-2 \alpha_{R}(t)} f_{\mathcal{R}}(\bar{x}, t)
$$

where $f_{\mathcal{R}}$ is interpreted as the parton density of the reggeon.

The question whether factorisation of hard physics and Regge factorisation, as expressed by (18), both be valid is not clear [21]. However, we find that in the analogous situation in $\left(\phi^{3}\right)_{6}$ perturbation theory, the dominant 1-loop diagrams as $z \rightarrow 1$ have a similar form to that in eq. (18), with a single propagator in place of the Regge pole.

\section{Cut Vertices}

In this part, we define space-like cut vertices $[6,7,8,9,10]$ and show that they may be identified with local operator matrix elements for inclusive DIS. We then extend the

\footnotetext{
${ }^{2}$ Note that extended fracture functions, which in the literature are also referred to as diffractive parton densities [20], are distinct from fracture functions, which do not depend on $t$. The latter are required when considering $d^{3} \sigma / d Q^{2} d z d x$ rather than $d^{4} \sigma / d Q^{2} d t d z d x$. In that case, one is not able to separate target and current fragmentation regions; a telling symptom is that the renormalisation group equation of an ordinary fracture function is not homogeneous $[14,15]$.

${ }^{3}$ The justification for the factorisation of the hard physics from the non-perturbative physics lies with factorisation theorems which we do not discuss here [20, 22].
} 

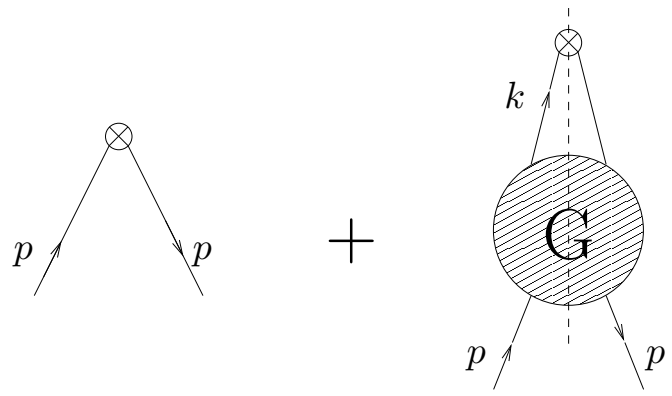

Figure 3: Definition of cut vertex.

argument to the semi-inclusive case, by defining generalised space-like cut vertices, and deriving the conditions under which they can be related to Green functions of local operators.

For the inclusive case, the lowest twist, $\operatorname{spin} j$ cut vertex ${ }^{4}$ is: [fig. 3]

$$
V_{\text {cut }}(p)=\left(p_{+}\right)^{j}+\int \frac{d^{d} k}{(2 \pi)^{d}} \Theta\left(0<k_{+}<p_{+}\right)\left(k_{+}\right)^{j} \underset{(k-p)^{2}}{\operatorname{disc}} G(k, p),
$$

where

$$
\begin{aligned}
& G(k, p)= \\
& \quad\left(\Delta_{F}(p)\right)^{-2} \int d^{d} x d^{d} y d^{d} z e^{i p \cdot x-i k \cdot(y-z)}\langle 0|T(\phi(x) \phi(y) \phi(z) \phi(0))| 0\rangle .
\end{aligned}
$$

The first term in (19) is the bare vertex, symbolised by $\otimes$, having two legs and Feynman rule $\left(k_{+}\right)^{j}$, where $k_{\mu}$ is the momentum passing through it. The second term is a convolution wrt. a loop momentum of the bare vertex and a cut four-point function. The thetafunction is encoded in the Feynman rules by letting the broken line of the cut four-point function pass through the $\otimes$.

The cut vertex is identical to the corresponding matrix element, [fig. 4a] defined as

$$
\begin{aligned}
& V_{\text {uncut }}(p)=\left\langle p\left|: \phi\left(-i \partial_{+}\right)^{j} \phi:(0)\right| p\right\rangle= \\
& \quad\left(\Delta_{F}(p)\right)^{-2} \int \frac{d^{d} k}{(2 \pi)^{d}}\left(k_{+}\right)^{j}\langle 0|T(\phi(p) \phi(-k) \phi(k) \phi(-p))| 0\rangle_{\text {disconn. }}
\end{aligned}
$$

where the "disconn." label is an instruction to keep connected parts and only those disconnected parts linked by the bare vertex. Thus,

$$
V_{\text {uncut }}(p)=\left(p_{+}\right)^{j}+\Delta_{F}^{-2}(p) \int \frac{d^{d} k}{(2 \pi)^{d}}\left(k_{+}\right)^{j} G(k, p) .
$$

\footnotetext{
${ }^{4}$ We require only the + light-cone components of the derivatives, since the rest are projected out by the Wilson co-efficients. Our conventions are $x_{ \pm}=\frac{1}{\sqrt{2}}\left(x_{0} \pm x_{3}\right), \partial_{ \pm} x_{\mp}=1$.
} 


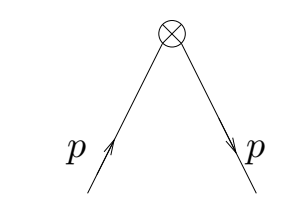

(a)

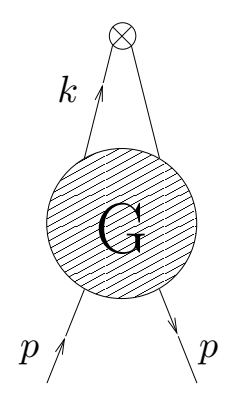

(b)

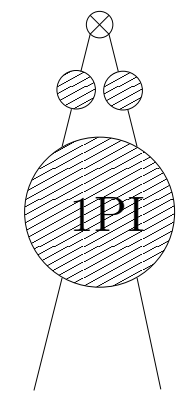

Figure 4: (a) Definition of uncut vertex. (b) Decomposition of second term in (a) in terms of two-point functions and 1PI four-point function.

To show that $V_{\text {uncut }}=V_{\text {cut }}$, we appeal to an argument of Baulieu et al.: [8] We note that $G(k, p)$ is not 1 PI wrt. the two legs carrying momentum $k$, and so has three discontinuities depending on $k$, two in the variable $k^{2}$ and one in $(k-p)^{2}$. In terms of 1PI Green functions, the uncut vertex is as in [fig. 4b]. We can parametrise these branch cuts individually by

$$
\text { (A) }\left(\int_{s_{0}}^{\infty} d \mu(s) \frac{1}{k^{2}-s+i \epsilon}\right)^{2}, \quad(B) \quad \int_{t_{0}}^{\infty} d \mu(t) \frac{1}{(k-p)^{2}-t+i \epsilon} .
$$

The branch cuts lie along

$$
\text { (A) } k^{2}=s-i \epsilon, \quad(B)(k-p)^{2}=t-i \epsilon,
$$

with $s, t>s_{0}, t_{0}$ respectively. Now consider these regions in the complex $k_{-}$plane. Letting $k^{2}=2 k_{-} k_{+}-k_{\perp}^{2}$ etc. and choosing $p_{\perp}=0$, we get

$$
\text { (A) } k_{-}=\frac{k_{\perp}^{2}+s-i \epsilon}{2 k_{+}}, \quad \text { (B) } k_{-}=p_{-}+\frac{k_{\perp}^{2}+t-i \epsilon}{2\left(k_{+}-p_{+}\right)} .
$$

The directions of the cuts depend on the sign of $k_{+}$and $(k-p)_{+}$. [fig. 5] For $0<k_{+}<p_{+}$ and $p_{+}<k_{+}<0$, the branch cuts lie on opposite sides of the imaginary axis, and there exists a contour deformation, $\gamma^{\prime}$, which wraps around the branch cut (B) in [fig. 6]. For the physical case of $p_{+}>0$, we only get a non-zero contribution from the discontinuity of $G$ wrt. $(k-p)^{2}$ in the interval $0<k_{+}<p_{+}$, as claimed.

Now we look at the semi-inclusive generalised cut vertices, which give us the $j$ th moments of the fracture functions, defined at lowest twist, spin $j$ [fig. 7] as

$$
\begin{aligned}
& V_{\text {cut }}\left(p, p^{\prime}\right)=\left(p_{+}-p^{\prime}{ }_{+}\right)^{j}\left|\Lambda\left(p, p^{\prime}\right)\right|^{2}+ \\
& \int \frac{d^{d} k}{(2 \pi)^{d}} \Theta\left(0<k_{+}<p_{+}-p^{\prime}{ }_{+}\right)\left(k_{+}\right)^{j} \underset{\left(k-p+p^{\prime}\right)^{2}}{\operatorname{disc}} G\left(k, p, p^{\prime}\right),
\end{aligned}
$$

where

$$
\Lambda\left(p, p^{\prime}\right)=\Delta_{F}^{-1}(p) \Delta_{F}^{-1}\left(p^{\prime}\right)\left\langle 0\left|T\left(\phi(p) \phi\left(-p^{\prime}\right) \phi\left(-p+p^{\prime}\right)\right)\right| 0\right\rangle
$$




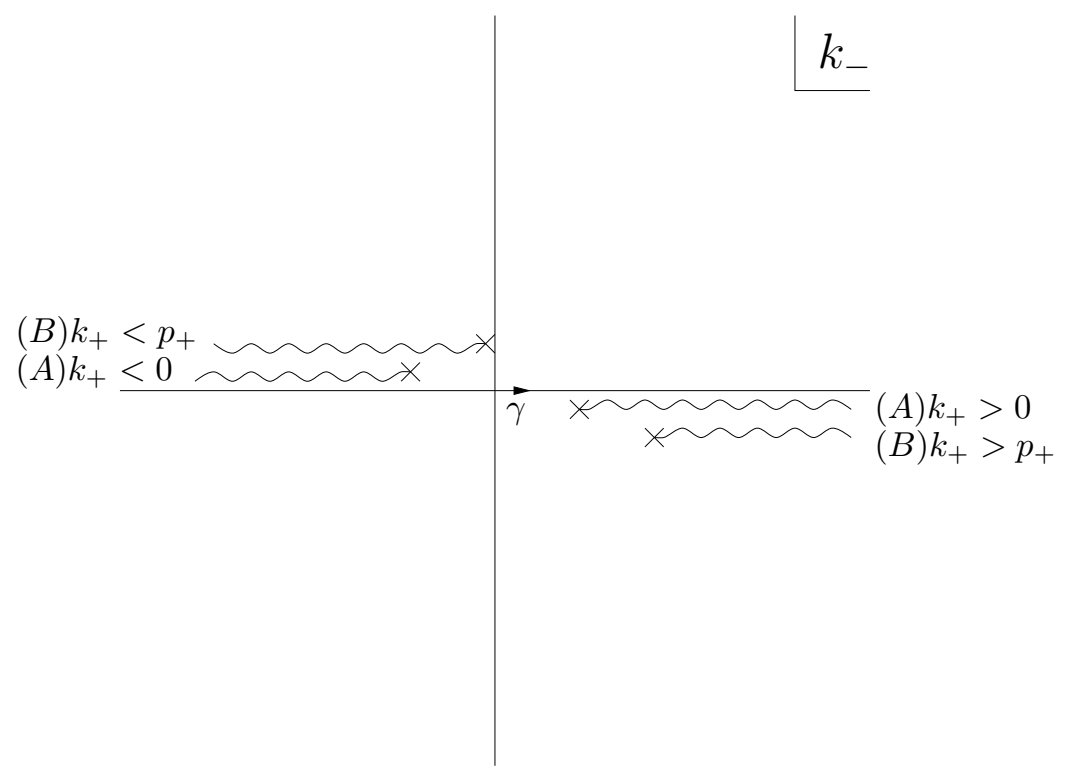

Figure 5: Pole structure of uncut vertex in $k_{-}$plane.

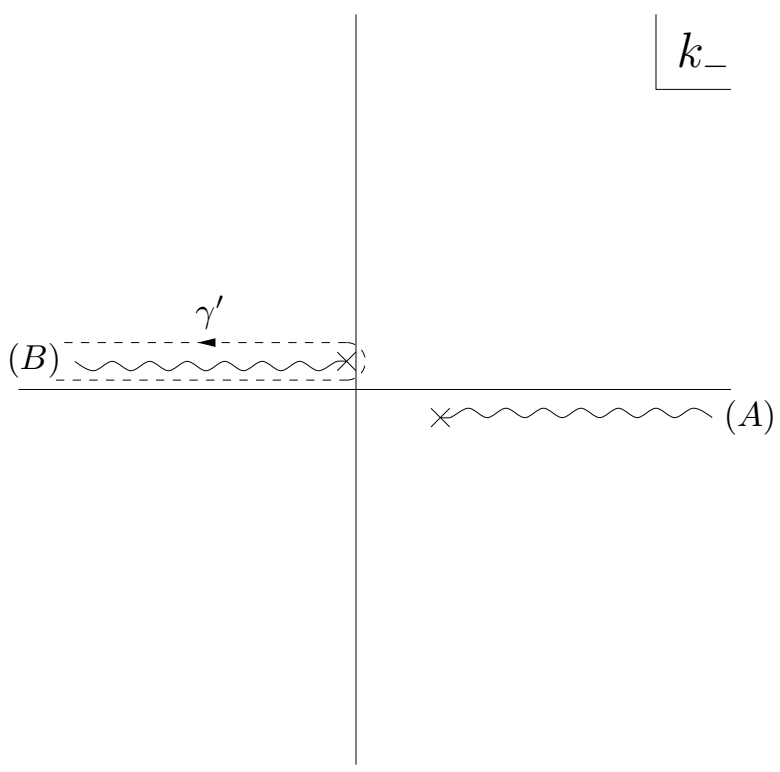

Figure 6: Contour deformation when $0<k_{+}<p_{+}$. 

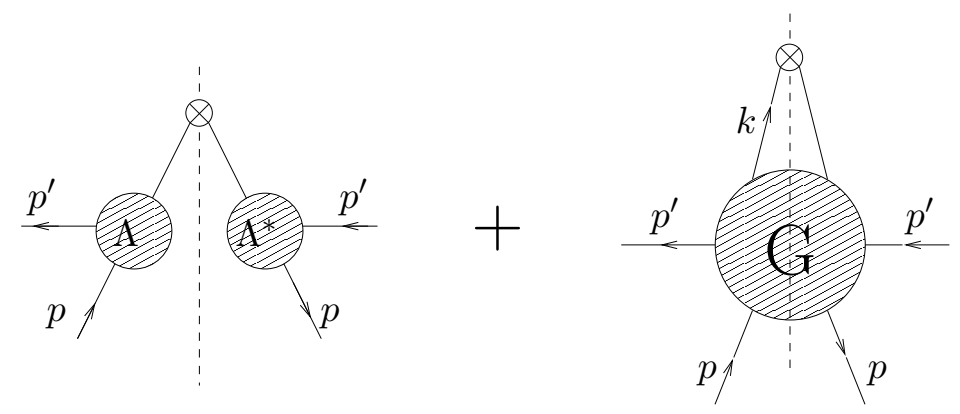

Figure 7: Definition of generalised cut vertex.
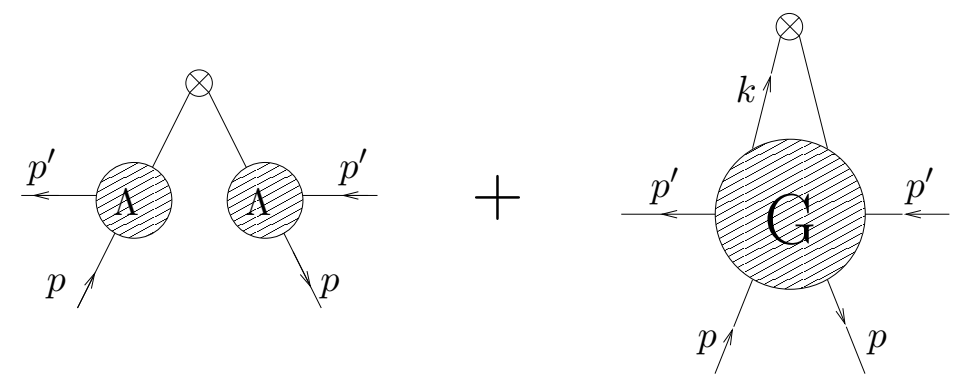

Figure 8: Definition of generalised uncut vertex.

and

$$
\begin{aligned}
G\left(k, p, p^{\prime}\right)= & \\
& \Delta_{F}^{-2}(p) \Delta_{F}^{-2}\left(p^{\prime}\right)\left\langle 0\left|T\left(\phi(p) \phi\left(-p^{\prime}\right) \phi(-k) \phi(k) \phi\left(p^{\prime}\right) \phi(-p)\right)\right| 0\right\rangle .
\end{aligned}
$$

The first term in (26) replaces the bare vertex of the previous case. We can also define an uncut vertex as follows:

$$
V_{\text {uncut }}\left(p, p^{\prime}\right)=\left(p_{+}-p_{+}^{\prime}\right)^{j} \Lambda^{2}\left(p, p^{\prime}\right)+\int \frac{d^{d} k}{(2 \pi)^{d}}\left(k_{+}\right)^{j} G\left(k, p, p^{\prime}\right) .
$$

$\Lambda^{2}=|\Lambda|^{2}$, so the first terms in (26) \& (29) are the same. The second terms are not the same, however, because $G\left(k, p, p^{\prime}\right)$ has a more complicated pole structure; it has branch cuts in $(\mathrm{A}) k^{2},(\mathrm{~B})(k-p)^{2},(\mathrm{C})\left(k+p^{\prime}\right)^{2}$ and $(\mathrm{D})\left(k-p+p^{\prime}\right)^{2}$ and we find it impossible to deform the $k_{-}$contour around just one of these. Putting $p^{\prime}{ }_{+}=z p_{+}+O\left(t / Q^{2}\right)$, we find the pole structure as in [fig. 9]. If we restrict $p_{+}>0$ and $z>0$, the contour deformations are as follows: 


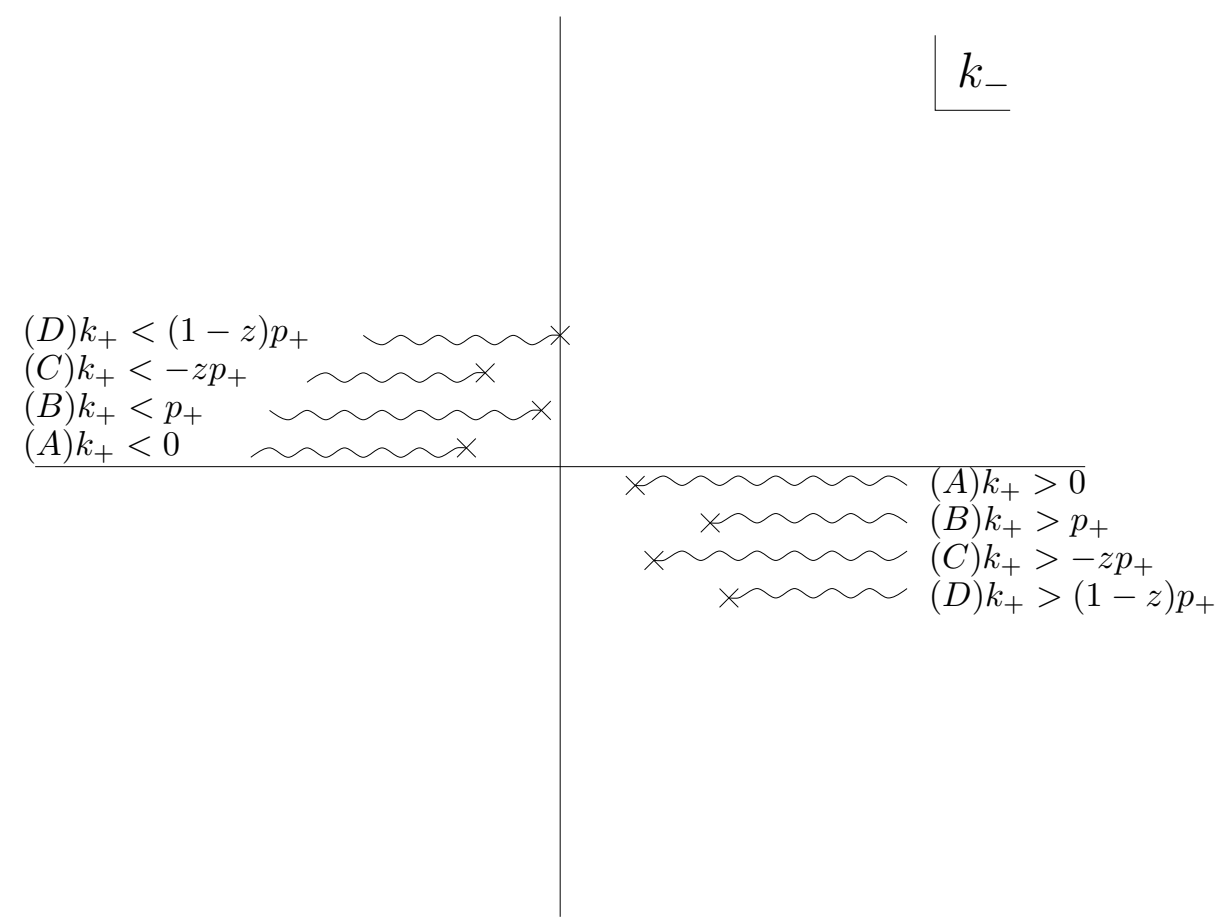

Figure 9: Pole structure of generalised uncut vertex in $k_{-}$plane.

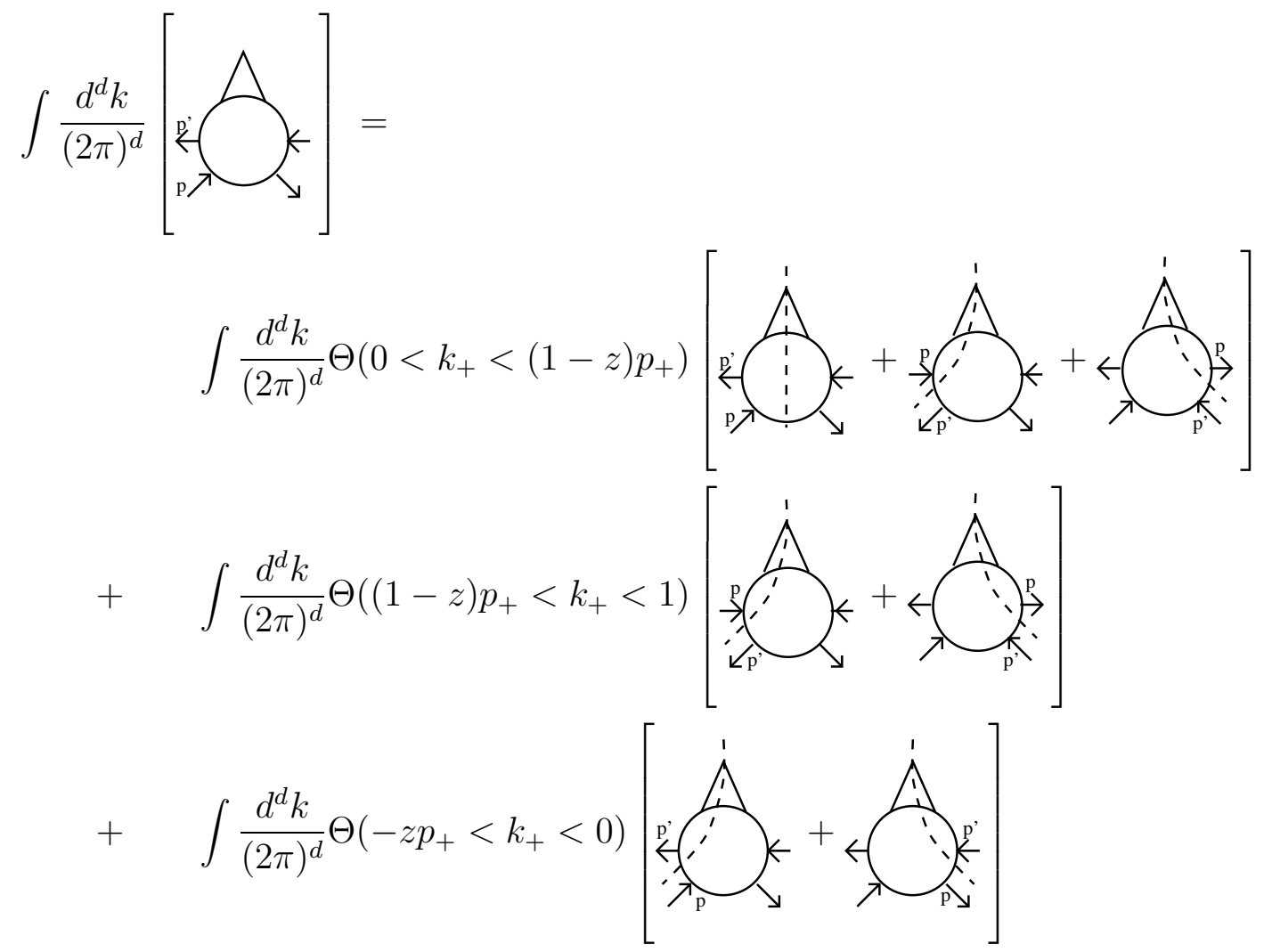




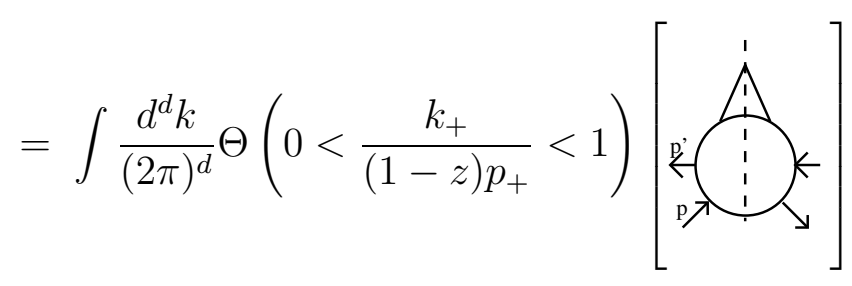

$$
\begin{aligned}
& +\int \frac{d^{d} k}{(2 \pi)^{d}} \Theta\left(-1<\frac{k_{+}}{z p_{+}}<0\right)[\underbrace{\mathrm{p}}_{-1} \\
& +\int \frac{d^{d} k}{(2 \pi)^{d}} \Theta\left(0<\frac{k_{+}}{p_{+}}<1\right)[\underbrace{}_{\gamma_{\mathrm{p}}}
\end{aligned}
$$

Eq. (30) is one of the main results of this paper. Expressed generally, it is that the Green function of a (lowest twist) composite operator is equal to the sum of all possible cuts between the other external legs.

The first term in eq. (30) is the generalised cut vertex, with the physical cut down the centre of the blob, but it is joined by other contributions. Moreover, the theta-function interval $0<k_{+}<(1-z) p_{+}$vanishes as $z \rightarrow 1$; together with the factor $\left(k_{+}\right)^{j}$ in the integrand, this means that the physical cut vanishes at least as fast as $(1-z)^{j}$. However, there is a class of diagrams which do not have the other cuts. These uncut diagrams can be identified with the physical cut down the centre, as we will see in section 5. For example, the Feynman diagram in [fig. 11f] can only be cut down the centre, giving a contribution to the generalised cut vertex. This class turns out to contain the dominant diagrams in the $z \rightarrow 1$ limit.

\section{Explicit Calculations in $\left(\phi^{3}\right)_{6}$ Theory}

To see what happens to the perturbative expansion of the generalised cut vertex as $z \rightarrow 1$, we present the results of calculations to one loop. These are in agreement with earlier calculations [23], which compute the cut vertices indirectly. We neglect contributions suppressed by powers of $t / Q^{2}$. It is found that the limit $z \rightarrow 1$ can be taken, leaving as dominant contributions only those diagrams where the $p$ and $p^{\prime}$ legs are connected to the rest of the diagram by single propagators. 


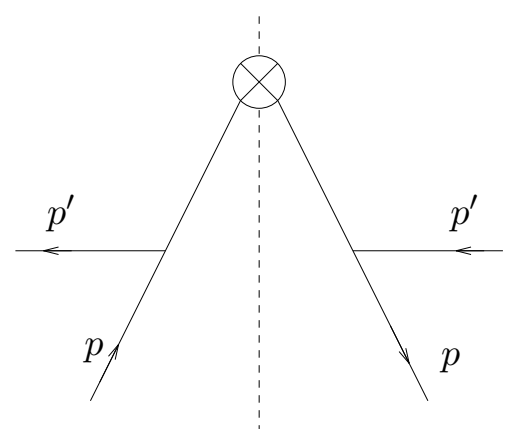

Figure 10: Tree-level cut vertex

To tree level, there is just one diagram, [fig. 10] given by

$$
V_{\mathrm{cut}}^{(0)}=\frac{\lambda^{2}}{t^{2}} p_{+}^{j}(1-z)^{j}
$$

where $\lambda$ is the bare coupling constant. The factor $(1-z)^{j}$ appears in all the diagrams and gives the leading order in $(1-z)$.

To one-loop order, there are eight diagrams [figs. 11a-h] and also their mirror images if they are not symmetric about the dotted line. [Figs. 11a\&b] are collinearly divergent, while [figs. 11f-h] are UV divergent. The rest are finite. Calculating in the $\overline{\mathrm{MS}}$ scheme, both types of divergence appear as simple poles in $\epsilon$, where the space-time dimension is $d=6-2 \epsilon$.

The diagrams are evaluated by choosing a frame where, in light-cone co-ordinates,

$$
\begin{aligned}
& p=\left(p_{+}, 0,0_{\perp}\right), \\
& q=\left(-x p_{+}, q_{-}, 0_{\perp}\right), \\
& p^{\prime}=\left(\alpha^{\prime} p_{+}, \beta^{\prime} q_{-}, p^{\prime}{ }_{\perp}\right), \\
& k=\left(\alpha p_{+}, \beta q_{-}, k_{\perp}\right),
\end{aligned}
$$

with

$$
\begin{aligned}
& \alpha^{\prime}=z+O\left(t / Q^{2}\right), \\
& \beta^{\prime} s=-t, \\
& p^{\prime 2}=-z t\left(1+O\left(t / Q^{2}\right)\right), \\
& s=2 p \cdot q .
\end{aligned}
$$

Thus, for example, the diagram in [fig. 11a] is

$$
\begin{aligned}
V_{\mathrm{cut}}^{(1 a)}= & \mu^{2 \epsilon} \lambda^{4} \int \frac{d^{d} k}{(2 \pi)^{d}} \frac{2 \pi \delta\left(\left(k-p+p^{\prime}\right)^{2}\right) \Theta\left(0<k_{+}<p_{+}-p_{+}{ }_{+}\right) k_{+}^{j}}{k^{4}\left(k+p^{\prime}\right)^{4}} \\
= & \frac{\mu^{2 \epsilon} \lambda^{4} p_{+}^{j} s}{4 \pi} \int_{0}^{1-z} d \alpha \alpha^{j} \int d \beta \int \frac{d^{d-2} k_{\perp}}{(2 \pi)^{d-2}} \\
& \times \frac{\delta\left((\alpha+z-1)(\beta s-t)-\left(k_{\perp}+p_{\perp}^{\prime}\right)^{2}\right)}{\left(\alpha \beta s-k_{\perp}^{2}\right)^{2}\left((\alpha+z)(\beta s-t)-\left(k_{\perp}+p_{\perp}^{\prime}\right)^{2}\right)^{2}}
\end{aligned}
$$


(a)

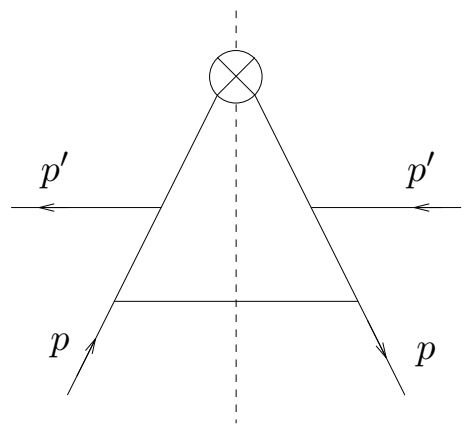

(c)

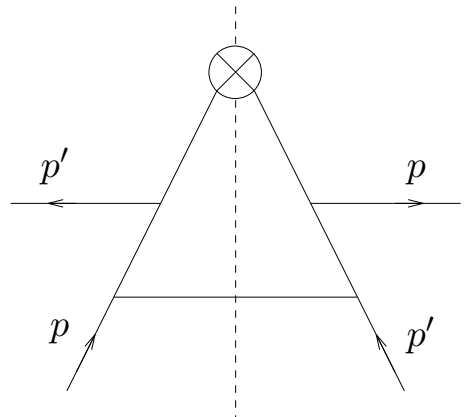

(e)

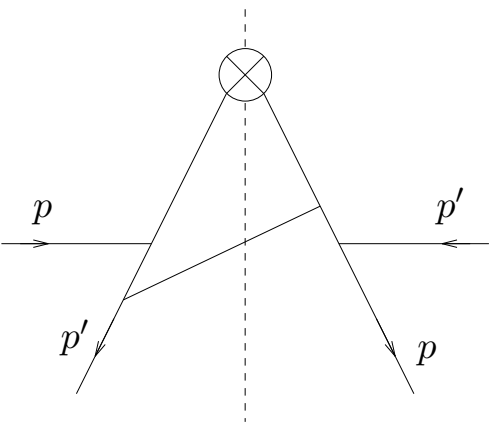

(g)

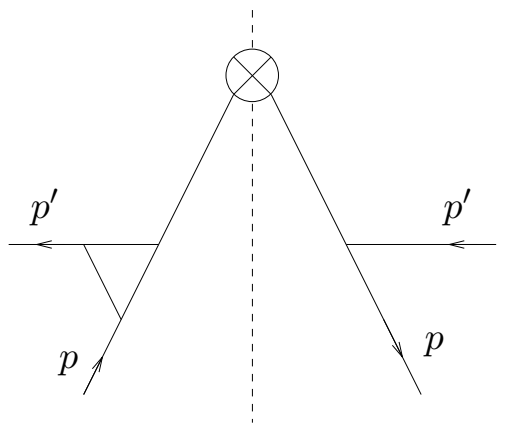

(b)

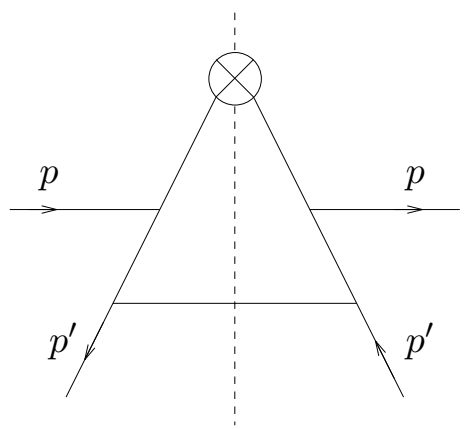

(d)

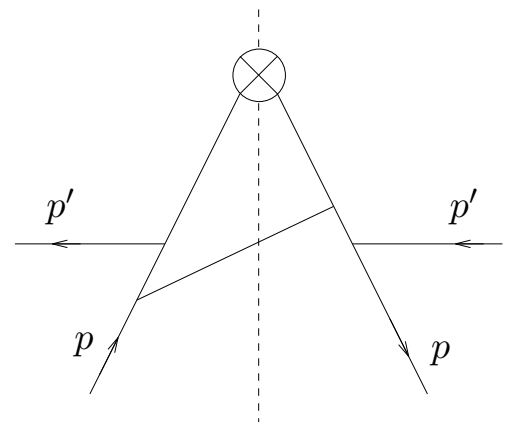

(f)

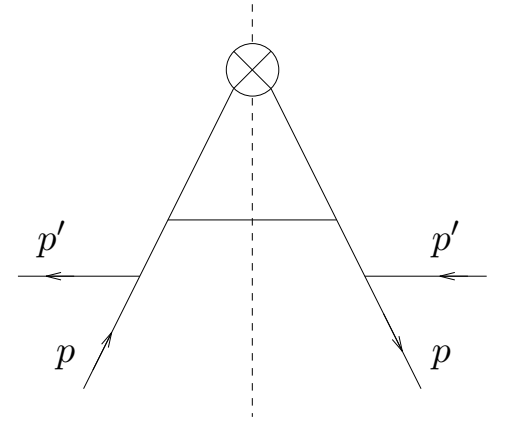

(h)

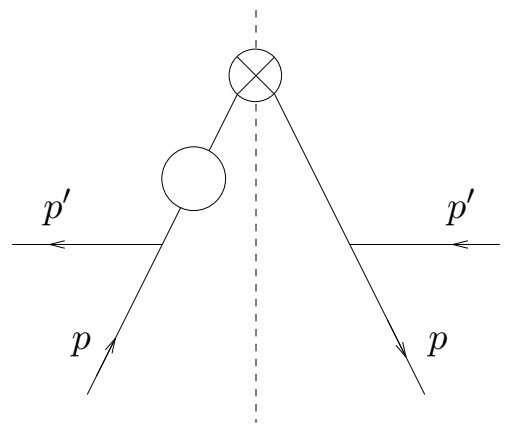

Figure 11: One-loop cut vertices 


$$
\begin{gathered}
=\frac{\lambda^{4}}{(4 \pi)^{3} t^{2}} p_{+}^{j}(1-z)^{j+2} \int_{0}^{1} \frac{d \alpha \alpha^{j}(1-\alpha)}{(\alpha+z(1-\alpha))^{2}}\left[-\frac{1}{\epsilon}\left(\frac{4 \pi \mu^{2}}{-t}\right)^{\epsilon} \Gamma(2+\epsilon)\right. \\
+2 \ln (\alpha+z(1-\alpha))+\ln (1-\alpha)-\ln \alpha-1] .
\end{gathered}
$$

Likewise, the other diagrams give, multiplying non-symmetric diagrams by 2 ,

$$
\begin{aligned}
V_{\text {cut }}^{(1 b)}= & \frac{\lambda^{4}}{(4 \pi)^{3} t^{2}} p_{+}^{j}(1-z)^{j+2} \int_{0}^{1} \frac{d \alpha \alpha^{j}(1-\alpha)}{(1-\alpha(1-z))^{2}}\left[-\frac{1}{\epsilon}\left(\frac{4 \pi \mu^{2}}{-t}\right)^{\epsilon} \Gamma(2+\epsilon)\right. \\
& -2 \ln z+\ln (1-\alpha)+2 \ln (1-\alpha(1-z))-\ln \alpha-1], \\
V_{\text {cut }}^{(1 c)}= & 2 \times \frac{\lambda^{4}}{2(4 \pi)^{3} t^{2}} p_{+}^{j} \frac{(1-z)^{j+2}}{z} \int_{0}^{1} d \alpha \alpha^{j}(1-\alpha)[4 \ln (1-z) \\
& +\ln \left(\frac{\alpha(1-\alpha)}{(1-(1-z)(1-\alpha))^{2}}\right)+\ln \left(\frac{\alpha(1-\alpha)}{\left.\left.(z+(1-z)(1-\alpha))^{2}\right)\right],}\right) \\
V_{\text {cut }}^{(1 d)}= & 2 \times \frac{\lambda^{4}}{(4 \pi)^{3} t^{2}} p_{+}^{j} \frac{(1-z)^{j+1}}{z} \int_{0}^{1} d \alpha \alpha^{j}[\ln (\alpha+z(1-\alpha))-\ln \alpha], \\
V_{\text {cut }}^{(1 e)}= & 2 \times \frac{\lambda^{4}}{(4 \pi)^{3} t^{2}} p_{+}^{j}(1-z)^{j+1} \int_{0}^{1} d \alpha \alpha^{j}[\ln z+\ln \alpha-\ln (1-\alpha(1-z))], \\
V_{\mathrm{cut}}^{(1 f)} & =\frac{\lambda^{4}}{(4 \pi)^{3} t^{2}} p_{+}^{j}(1-z)^{j} \frac{1}{\epsilon}\left(\frac{4 \pi \mu^{2}}{-t}\right)^{\epsilon} \int_{0}^{1} d \alpha \alpha^{j}(1-\alpha) .
\end{aligned}
$$

The rest are straightforward virtual loop corrections giving

$$
\begin{gathered}
V_{\mathrm{cut}}^{(1 g)}=2 \times \frac{\lambda^{4}}{2(4 \pi)^{3} t^{2}} p_{+}^{j}(1-z)^{j} \frac{1}{\epsilon}\left(\frac{4 \pi \mu^{2}}{-t}\right)^{\epsilon}, \\
V_{\mathrm{cut}}^{(1 h)}=2 \times-\frac{\lambda^{4}}{12(4 \pi)^{3} t^{2}} p_{+}^{j}(1-z)^{j} \frac{1}{\epsilon}\left(\frac{4 \pi \mu^{2}}{-t}\right)^{\epsilon} .
\end{gathered}
$$

Taking the limit $z \rightarrow 1$ and, keeping only leading orders in $(1-z)$, we have

$$
\begin{gathered}
V_{\text {cut }}^{(1 a)}=\frac{\lambda^{4}}{(4 \pi)^{3} t^{2}} p_{+}^{j}(1-z)^{j+2} \frac{1}{(j+1)(j+2)}\left[-\frac{1}{\epsilon}\left(\frac{4 \pi \mu^{2}}{-t}\right)^{\epsilon} \Gamma(2+\epsilon)\right. \\
\left.-\gamma_{E}-\psi(j+1)\right]+O\left((1-z)^{j+3}\right),
\end{gathered}
$$




$$
\begin{aligned}
& V_{\text {cut }}^{(1 b)}=V_{\text {cut }}^{(1 a)}+O\left((1-z)^{j+3}\right), \\
& V_{\text {cut }}^{(1 c)}=2 \times-\frac{\lambda^{4}}{(4 \pi)^{3} t^{2}} p_{+}^{j}(1-z)^{j+2} \frac{1}{(j+1)(j+2)} \\
& \times\left[2 \ln \left(\frac{1}{1-z}\right)-1+\gamma_{E}+2 \psi(j+3)-\psi(j+1)\right] \\
& +O\left(\ln \left(\frac{1}{1-z}\right)(1-z)^{j+3}\right) \text {, } \\
& V_{\text {cut }}^{(1 d)}=2 \times \frac{\lambda^{4}}{(4 \pi)^{3} t^{2}} p_{+}^{j}(1-z)^{j+1} \frac{1}{(j+1)^{2}}+O\left((1-z)^{j+2}\right), \\
& V_{\text {cut }}^{(1 e)}=2 \times-\frac{\lambda^{4}}{(4 \pi)^{3} t^{2}} p_{+}^{j}(1-z)^{j+1} \frac{1}{(j+1)^{2}}+O\left((1-z)^{j+2}\right), \\
& V_{\text {cut }}^{(1 f)}=\frac{\lambda^{4}}{(4 \pi)^{3} t^{2}} p_{+}^{j}(1-z)^{j} \frac{1}{\epsilon}\left(\frac{4 \pi \mu^{2}}{-t}\right)^{\epsilon} \frac{1}{(j+1)(j+2)}, \\
& V_{\mathrm{cut}}^{(1 g)}=2 \times \frac{\lambda^{4}}{2(4 \pi)^{3} t^{2}} p_{+}^{j}(1-z)^{j} \frac{1}{\epsilon}\left(\frac{4 \pi \mu^{2}}{-t}\right)^{\epsilon}, \\
& V_{\text {cut }}^{(1 h)}=2 \times-\frac{\lambda^{4}}{12(4 \pi)^{3} t^{2}} p_{+}^{j}(1-z)^{j} \frac{1}{\epsilon}\left(\frac{4 \pi \mu^{2}}{-t}\right)^{\epsilon} .
\end{aligned}
$$

In the above, finite parts proportional to $p_{+}^{j}(1-z)^{j} / t^{2}$ are neglected. To renormalise the UV poles of [figs. 11f-h], we introduce the running coupling constant $\lambda(t)$ in terms of the bare coupling $\lambda$,

$$
\lambda^{2}=\lambda^{2}(t)\left(1-\frac{3}{4} \frac{\lambda^{2}}{(4 \pi)^{3}} \frac{1}{\epsilon}\left(\frac{4 \pi \mu^{2}}{-t}\right)^{\epsilon}\right),
$$

and multiply by a renormalisation factor $Z_{\phi}^{-2} Z_{j}, Z_{j}$ being for the spin $j$ bare vertex, such that $^{5}$

$$
Z_{\phi}=1+\frac{1}{12} \frac{1}{\epsilon} \frac{\lambda^{2}}{(4 \pi)^{3}}
$$

and

$$
Z_{j}=1-P_{j} \frac{1}{\epsilon} \frac{\lambda^{2}}{(4 \pi)^{3}}
$$

\footnotetext{
${ }^{5}$ Here, the renormalisation prescription differs from that of ref. [23], which simply introduces the running coupling.
} 

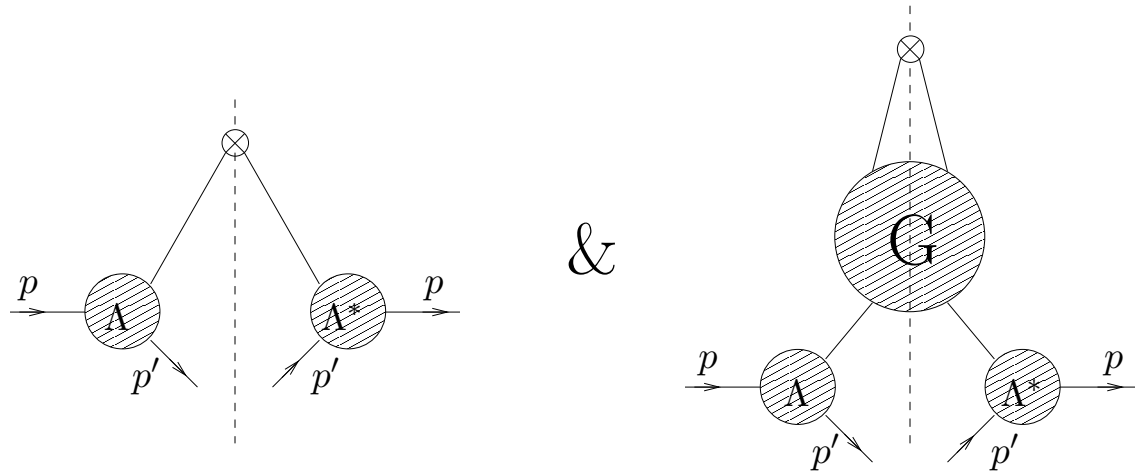

Figure 12: Topology of the dominant one-loop diagrams.

where $P_{j}$ is the $j$ th moment of the AP kernel for $\left(\phi^{3}\right)_{6}$ theory, $P(x)=x(1-x)-\frac{1}{12} \delta(1-x)$ :

$$
P_{j}=\int_{0}^{1} \frac{d x}{x} x^{j} P(x)=\frac{1}{(j+1)(j+2)}-\frac{1}{12} .
$$

The final result, to order $(1-z)^{j}$, is

$$
\begin{aligned}
V_{\text {cut,ren. }}^{1-\text { loop }}\left(p, p^{\prime}\right)= & \frac{\lambda^{2}(t)}{t^{2}} p_{+}^{j}(1-z)^{j}\left[1+\frac{\lambda^{2}}{(4 \pi)^{3}} \ln \left(\frac{4 \pi \mu^{2}}{-t}\right)\left(\frac{1}{6}+P_{j}\right)\right] \\
& +O\left(\ln \left(\frac{1}{1-z}\right)(1-z)^{j+2}\right) .
\end{aligned}
$$

The observation we make is that only diagrams in [figs. 10\&11f-h] contribute to leading order in $(1-z)$.

The diagrams in [figs. 10\&11f-h] have the topology of [fig. 12], expressible as

$$
\begin{aligned}
& V_{\text {cut }}\left(p, p^{\prime}\right) \stackrel{z \rightarrow 1}{\longrightarrow}\left[\left(p_{+}-p_{+}^{\prime}\right)^{j}+\right. \\
& \left.\quad \int \frac{d^{d} k}{(2 \pi)^{d}} \Theta\left(0<k_{+}<p_{+}-p_{+}^{\prime}\right)\left(k_{+}\right)^{j} \underset{\left(k-p+p^{\prime}\right)^{2}}{\operatorname{disc}} G\left(k, p-p^{\prime}\right)\right]\left|\Lambda\left(p, p^{\prime}\right)\right|^{2},
\end{aligned}
$$

with $G$ and $\Lambda$ as defined in (20)\&(27). Note that $G$ is collinear-safe to one-loop since the lines carrying $p-p^{\prime}$ are off shell by $t$. Now, recall that in section 4 it was shown that uncut diagrams were equal to a sum of all possible cuts between external legs. For the dominant diagrams, the only cuts are the ones contributing to $V_{\text {cut }}\left(p, p^{\prime}\right)$; eq. (55) may be equated with its uncut analogue:

$$
\left[\left(p_{+}-p_{+}^{\prime}\right)^{j}+\int \frac{d^{d} k}{(2 \pi)^{d}}\left(k_{+}\right)^{j} G\left(k, p-p^{\prime}\right)\right] \Lambda^{2}\left(p, p^{\prime}\right)
$$




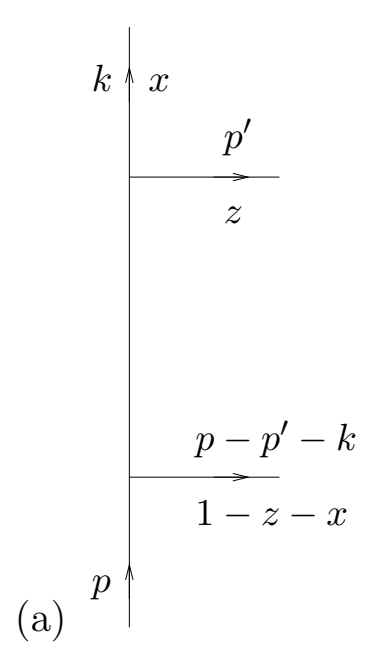

Figure 13: Matrix elements corresponding to (a) fig. 11a \& (b) fig. 11f. The external legs are marked with 4 -momenta and light-cone components scaled by $p_{+}$, such that $k_{+}=x p_{+}$ and $p_{+}^{\prime}=z p_{+}$.

Thus, we find that at small $(1-z)$ the generalised cut vertices depend approximately on the Green function of a composite operator.

\section{Discussion \& Conclusions}

The main conclusion of sections $4-5$ is the following: If the dominating contribution to the fracture function has the topology of [fig. 12], then the fracture function can be identified with an uncut amplitude. Now we discuss whether this is the case for QCD.

Although perturbative calculations are not appropriate to describe the process in question, they can give insight into certain features of the process which can be expected to be independent of non-perturbative effects. The dominance of a particular class of processes as $z \rightarrow 1$ is one of them. First, we give a simple argument to explain the $(1-z)$ dominance of this class of process in the perturbative expansion, and compare it with another perturbative model which has been studied by Hautmann et al. [24]. Secondly, we discuss the Regge hypothesis of ref. [5].

Let us contrast the behaviour of the diagrams in [figs. 11a\&f], the first of which is suppressed in $(1-z)$. These have matrix elements as in [figs. 13a\&b]. In (b), the propagator connecting the $p^{\prime}$ leg and the $\left(p-p^{\prime}-k\right)$ leg is fixed and off shell by $t$. In (a), the propagator is constrained by the condition that the $\left(p-p^{\prime}-k\right)$ leg be on shell and 


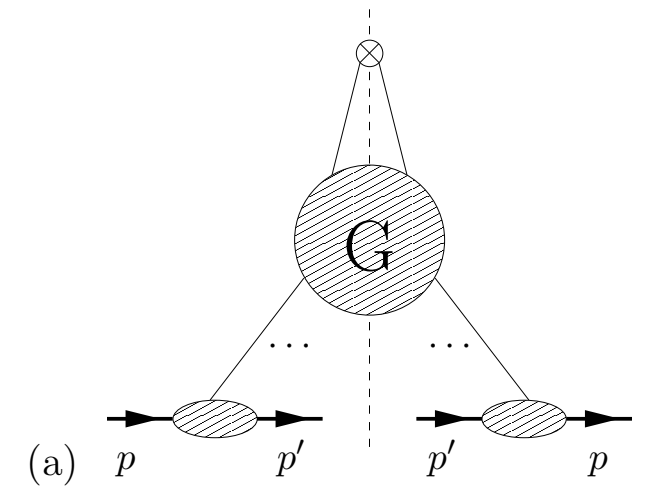

(b)

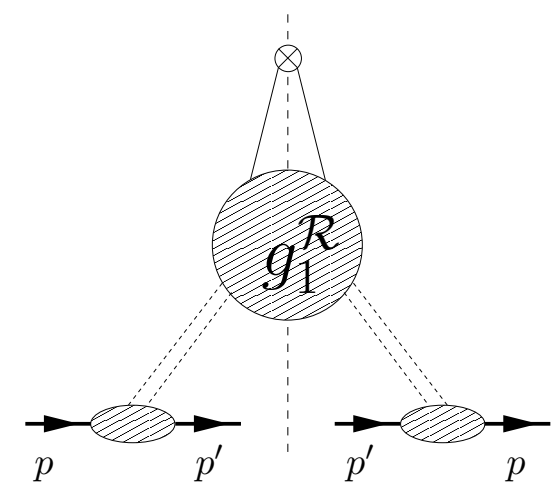

Figure 14: (a) Virtual exchanges between the bare vertex and the hadronic states in the $z \rightarrow 1$ limit. (b) $z \rightarrow 1$ limit of the polarised fracture function, with $g_{1}^{\mathcal{R}}$ the polarised reggeon structure function.

equals

$$
\frac{1}{\left(k+p^{\prime}\right)^{2}}=-\frac{(1-x-z)}{\left(k_{\perp}+p_{\perp}^{\prime}\right)^{2}} \sim \frac{1-z}{t},
$$

at small $k_{\perp}$. Diagrams in [fig. 11a-c] have two such propagators off shell by $\frac{t}{1-z}$ and are suppressed by $(1-z)^{2}$ as a result, while [fig. 11d-e] have one, and go like $(1-z)$ instead.

The rule appears to be that if the line carrying the momentum component $z p_{+}$branches out with soft emissions into the final state, its contribution is small. If this carries over into the non-perturbative physics, then the dominant class of processes is as in [fig. 14a]. Note: The factor $G$ is a connected, cut amplitude. If we tried to fit, say, [fig. 11a] into this topology, it would give a disconnected contribution to $G$, since there is a soft line (carrying $\left.\left(p-p^{\prime}-k\right)\right)$ going into the final state.

In a perturbative QCD heavy-quark model for such a process, Hautmann et al. [24] also find that such diagrams are dominant, with the single propagators replaced by gluon pairs in a colour singlet. In that case, $G$ can be defined in terms of cut Green functions, although these are not then equal to uncut ones: $G$ is connected to the hadronic states by more than one virtual line. However, this is only a difficulty in QCD perturbation theory because of the multiparticle poles. The Regge model proposed in ref. [5] provides a non-perturbative description of the virtual exchanges [fig. 14b]. Here, we expect the pole structure to be simple enough to uncut the amplitude in the same way as the $\phi^{3}$ diagrams: The reggeon structure function $g_{1}^{\mathcal{R}}$ is equal to a connected 3-point amplitude depending on an insertion of a local operator. It is possible to perform the decomposition of this amplitude into 1PI vertices and two-point functions, as outlined in section 2.

To summarise, we have shown that in the limit $z \rightarrow 1$, fracture functions are dominated by a special class of process. If a Regge model for this is realistic, these processes are related to an uncut amplitude. This would mean that that the polarised reggeon structure 
function can be used as a testing-ground for the target-independence hypothesis of the "proton spin effect".

\section{Acknowledgements}

We should like to thank Z. Kunszt, L. Trentadue and G. Veneziano for discussions during the course of this research. The work of M.G. is supported by the Swiss National Foundation. G.M.S. and B.E.W. are supported by PPARC grant GR/L56374 in the UK. G.M.S. acknowledges EC TMR Network Grant FMRX-CT96-0008. B.E.W. also wishes to thank PPARC for a research studentship.

\section{References}

[1] S. D. Bass, hep-ph/9902280 and references therein.

[2] G. M. Shore \& G. Veneziano, Nucl. Phys. B381 (1992) 23.

[3] S. Narison, G. M. Shore \& G. Veneziano, Nucl. Phys. B433 (1995) 209, hep-ph/9404277.

[4] S. Narison, G. M. Shore \& G. Veneziano, hep-ph/9812333, for an extended analysis with non-zero quark masses.

[5] G. M. Shore \& G. Veneziano, Nucl. Phys. B516 (1998) 333, hep-ph/9709213.

[6] A. H. Mueller, Phys. Rev. D 18 (1978) 3705.

[7] S. Gupta \& A. H. Mueller, Phys. Rev. D 20 (1979) 118.

[8] L. Baulieu, E. G. Floratos \& C. Kounnas, Nucl. Phys. B166 (1980) 321.

[9] T. Kubota, Nucl. Phys. B165 (1980) 277.

[10] T. Munehisa, Prog. Th. Phys. 67 (1982) 882.

[11] L. Trentadue \& G. Veneziano, Phys. Lett. 323B (1994) 201.

[12] D. Graudenz, Nucl. Phys. B432 (1994) 351.

[13] D. de Florian, \& R. Sassot, Phys. Rev D 56 (1997) 426, hep-ph/9703228.

[14] M. Grazzini, L. Trentadue \& G. Veneziano, Nucl. Phys. B519 (1998) 394, hep-ph/9709452. 
[15] G. Camici, M. Grazzini \& L. Trentadue, Phys. Lett. 439B (1998) 382, hep-ph/9802438.

[16] G. M. Shore, hep-ph/9812354, for an introduction to the $U_{A}(1)$ problem.

[17] G. Altarelli \& G. G. Ross, Phys. Lett. 212B (1988) 391.

[18] R. D. Ball, S. Forte \& G. Ridolfi, Phys. Lett. 378B (1996) 255, hep-ph/9510449.

[19] G. M. Shore, Nucl. Phys. B Proc. Suppl. 64 (1998) 167, hep-ph/9710367.

[20] A. Berera \& D. E. Soper, Phys. Rev. D 53 (1996) 6162 , hep-ph/9509239.

[21] A. Berera, Proceedings of the International Workshop on Deep-Inelastic Scattering and Related Topics, Rome, Italy 1996, ed. G. d'Agostini, hep-ph/9606448;

Z. Kunszt \& W. J. Stirling, hep-ph/9609245.

[22] M. Grazzini, Phys. Rev. D 57 (1998) 4352, hep-ph/9710272;

J. C. Collins, Phys. Rev. D 57 (1998) 3051, hep-ph/9709499.

[23] M. Grazzini, Nucl. Phys. B518 (1998) 303, hep-ph/9709312.

[24] F. Hautmann, Z. Kunszt \& D. E. Soper, Phys. Rev. Lett. 81 (1998) 3333, hep-ph/9806298. 